\title{
Rheumatologische medikamentöse Therapie bei Malignomanamnese
}

\section{Rheumatological Drug Treatment in Patients with a History of Malignancy}

\author{
Autor \\ Marc Schmalzing \\ Institut \\ Rheumatologie / Klinische Immunologie, Medizinische Klinik \\ und Poliklinik II, Universitätsklinikum Würzburg, Würzburg \\ Schlüsselwörter \\ Basistherapie, Malignom, Immunsuppression \\ Key words \\ DMARD, malignancy, immunosuppression \\ online publiziert $\quad 21.10 .2020$ \\ Bibliografie \\ Akt Rheumatol 2020; 45: 523-530 \\ DOI 10.1055/a-1247-4252 \\ ISSN 0341-051X \\ (C) 2020. Thieme. All rights reserved. \\ Georg Thieme Verlag KG, Rüdigerstraße 14, \\ 70469 Stuttgart, Germany \\ Korrespondenzadresse \\ Dr. med. Marc Schmalzing \\ Rheumatologie/ Klinische Immunologie \\ Medizinische Klinik und Poliklinik II \\ Universitätsklinikum Würzburg \\ Oberdürrbacher Straße 6 \\ 97080 Würzburg \\ Deutschland \\ Tel.: + 49931201 40100, Fax: + 490931201640100 \\ Schmalzing_m@ukw.de
}

\section{ZUSAMMENFASSUNG}

Rheumatologische Therapie bei Patienten mit Malignomanamnese ist mit komplexen Fragestellungen verbunden. Schwierigkeiten und Lösungsmöglichkeiten bei der Interpretation aussagekräftiger Studien werden dargestellt. Empfehlungen in Leitlinien zu diesem Thema werden diskutiert. Nationale Register und Versicherungsdatenbanken wurden mit der Frage nach Tumorrezidivrisiko unter Basistherapeutika untersucht ; diese Analysen beziehen sich aber v. a. auf TNF-Inhibitoren und Rituximab. Zu den gängigen Substanzen der Basistherapie werden Daten zur Tumorinzidenz und wenn vorhanden zum Tumorrezidivrisiko zusammengefasst. Abschließend wird der Versuch unternommen Vorschläge zur rheumatolgischen Therapie bei Malignomanamnese zu formulieren.

\section{ABSTRACT}

Rheumatological therapy in patients with a history of malignancy is associated with complex questions. Difficulties and possible solutions in the interpretation of meaningful studies are presented. Recommendations in guidelines on this topic are discussed. National registries and insurance databases have been examined as to the tumour recurrence risk under diseasemodifying antirheumatic drugs; however, these analyses mainly refer to TNF inhibitors and rituximab. Data on tumor incidence and, if available, tumor recurrence risk are summarized for common disease-modifying antirheumatic drugs. Finally, an attempt is made to formulate proposals for rheumatological therapy in patients with a history of malignancy.

\section{Einleitung}

Mit zunehmenden Alter der Bevölkerung steigt die Prävalenz maligner Erkrankungen. Diese sind mit hohem Leidensdruck, starken krankheitsbezogenen Ängsten und einem großen Therapiewunsch vergesellschaftet. Durch Entwicklung verträglicherer Chemosowie Strahlentherapieprotokolle, minimalinvasiver chirurgischer Techniken und nicht zuletzt von zielgerichteten oder immunonkologischen Therapieformen kann ein zunehmender Anteil dieser Patienten geheilt werden oder die maligne Erkrankung kann zumindest über einen langen Zeitraum im Sinne einer chronischen Er- krankung stabil gehalten werden mit hoher Lebensqualität für die Patienten.

Ähnliche Entwicklungen hat die Rheumatologie erfahren. Die konsequente leitliniengerechte Basistherapie rheumatisch-entzündlicher Erkrankungen wird zunehmend auch für ältere Patienten propagiert und es sind auch in Zukunft weitere Zulassungen für Krankheitsentitäten zu erwarten, die vornehmlich Ältere betreffen, wie die Riesenzellarteriitis. Die Zeiten sollten vorbei sein, in denen ältere Rheumakranke mangels Alternativen nur «ein bisschen» - meist zu viel - Glukokortikoide vom primär betreuenden 
Arzt erhalten. Differenziertere Therapien bedürfen natürlich auch einer engmaschigeren Therapieführung durch die Rheumatologin oder den Rheumatologen.

Vor diesem Hintergrund erscheint es logisch, dass man in der Rheumatologie zunehmend mit der Frage konfrontiert wird, wie die Therapie einer rheumatisch-entzündlichen Erkrankungen gestaltet werden sollte, wenn der Patient eine maligne Erkrankung hatte oder vielleicht sogar noch hat. Teilweise muss man hierbei erhebliche Sorgen und Vorbehalte von Patienten und mitbehandelnden Kollegen berücksichtigen.

\section{Zunehmende Datenfülle, schwierige Interpretation}

Um derartige therapeutische Entscheidungen treffen zu können ist es meist wenig hilfreich, sich mit der beeindruckenden und spannenden Datenfülle über in vitro-Experimente oder Tiermodelle zum Wechselspiel zwischen Tumorzellen und Immunsystem zu beschäftigen. Einerseits ist eine der Hauptaufgaben des Immunsystems die tumour surveillance, also die Erkennung und Zerstörung von malignen Zellen. Andererseits kann Entzündung aber auch zur Tumorproliferation oder zur Inhibierung von tumour surveillance beitragen. Chronische Entzündung erhöht bekanntermaßen das Lymphomrisiko [1]. Ähnliche Beobachtungen existieren auch für bestimmte solide Tumore abhängig vom Tumorstadium [2-4]. Dabei können dieselben Zellgruppen oder Zytokine abhängig vom Mikromilieu ganz unterschiedliche Wirkungen entfalten, also sowohl tumorinhibierend als auch tumorfördernd. So kann z. B.TNFalpha, das sonst v. a. als tumorinhibierendes Zytokin bekannt ist, bei Ovarialkarzinomen regulatorische T-Zellen stimulieren, die mit einem höheren Progressionsrisiko assoziiert sind, weil sie wahrscheinlich tumour surveillance unterdrücken [5].

Die Studien, die sonst in der Medizin die beste Evidenz liefern, nämlich randomisiert kontrollierte Studien, eignen sich selbst nach Metaanalyse nur sehr bedingt, um die Frage zu klären ob das jeweilige Präparat das Tumorrezidivrisiko erhöht. Dafür sind mehrere Gründe anzuführen:

- Die Fallzahlberechnung und das Design von Zulassungsstudien orientiert sich primär am Nachweis der Effektivität.

Dadurch sind die Fallzahlen zu klein und die Nachbeobachtung zu kurz um bestimmte Sicherheitsaspekte zu erfassen.

- Bei malignen Erkrankungen handelt es sich um seltene Ereignisse, die regelhaft erst nach langer Latenz auftreten. Unter Cyclophosphamid betragen diese Latenzen zwischen 10 und 15 Jahren.

- Maligne Vorerkrankungen (mit der Ausnahme von nichtmelanozytären Hautkrebsformen) und oft hohes Alter stellen Ausschlusskriterien dar. Auch weisen ältere Patienten nicht selten Komorbiditäten oder logistische Einschränkungen auf, sodass Patienten mit hohem Krebsrisiko in Studien meist unterrepräsentiert sind.

Es existieren relativ wenige Studien, die das Tumorrezidivrisiko unter immunsuppressiver Therapie primär zum Gegenstand haben. Dabei handelt es sich um Auswertungen nationaler Register oder von Versicherungsdatenbanken. Da die teilnehmenden Ärzte aber in diesen Studien selber festlegen, welche Therapie ein Patient mit maligner Vorerkrankung erhält, kann es zu Verfälschungen kommen, indem Präparate, denen der Arzt ein hohes tumorförderndes Potential zumisst, bei Patienten mit hohem Tumorrezidivrisiko zurückhaltender eingesetzt werden. Diesem Bias versucht man in der statistischen Analyse dieser Studien dadurch zu begegnen, dass man nach den üblichen Risikofaktoren für Tumorrezidive (z. B. das initiale Tumorstadium) adjustiert. Der Vorteil von Versicherungsdatenbanken ist die hohe Fallzahl, der Nachteil die wenig detaillierte Information zum einzelnen Patienten, was eine Adjustierung erheblich erschwert.

Mangels direkter Evidenz zum Tumorrezidivrisiko versucht man sich bei den meisten Präparaten am Risiko für das Auftreten von neuen, inzidenten Malignomen in randomisierten Studien, Registern und Versicherungsdatenbanken zu orientieren und extrapoliert dieses Risiko auf das Rezidivrisiko.

Angesichts dieser Einschränkungen in der Datenlage bieten sich darüber hinaus weitere Möglichkeiten des Erkenntnisgewinns an:

- Man betrachtet Studien zur immunsuppressiven Therapie bei Risikopopulationen, die eine höhere Wahrscheinlichkeit für maligne Erkrankungen haben: z. B. Patienten mit Präkanzerosen wie zervikalen intraepithelialen Neoplasien oder monoklonalen Gammopathien.

- Für Studien, in denen unterschiedliche Dosierungen verwendet werden, kann ein Dosiseffekt untersucht werden.

- Bei nicht-melanozytären Hautkrebsformen (NMSC) handelt es sich um maligne Erkrankungen, die häufiger auftreten und für die selbst bei Studien mit kürzerer Nachbeobachtung Unterschiede gesehen wurden [6]. Diese Entitäten könnten eingeschränkt als Surrogat für das Krebsrisiko insgesamt dienen, zumal sie meist kein Ausschlusskriterien für randomisiert-kontrollierte Studien darstellen. Allerdings werden NMSC selbst in Studien oft nicht komplett erfasst, was den Grund darstellt, warum sie häufig in Betrachtungen zum Malignitätsrisiko ausgenommen werden.

Bei der Betrachtung von Studien zur Häufigkeit von malignen Erkrankung gilt ein besonderes Augenmerk der jeweiligen Kontrollgruppe. Vergleiche mit der Normalbevölkerung sind weniger hilfreich, da rheumatisch-entzündliche Erkrankungen unabhängig von Einflüssen der Therapie oft erhöhte Inzidenzen für bestimmte maligne Erkrankungen aufweisen, wie z. B. das Bronchialkarzinom bei rheumatoider Arthritis [7], Lymphome bei Sjögren-Syndrom [8] und das Zervixkarzinom bei Systemischem Lupus erythematodes [9].

\section{Aussagen in Leitlinien und Empfehlungen}

Es existieren viele nationale und internationale Leitlinien zur Therapie der rheumatoiden Arthritis, die sich unterschiedlich detailliert mit dem Thema Malignomanamnese befassen [10]. Die klarsten Empfehlungen hierzu wurden 2012 und 2015 vom American College of Rheumatology formuliert [11, 12]. In den älteren Empfehlungen von 2012 wurde noch -wie in den meisten anderen Leitlinien - unterschieden, ob der Abstand zwischen Ende der kurativen Tumortherapie und Beginn der Basistherapie mehr oder höchstens 5 Jahre betrug. Dabei konnte man sich damals nur auf die übliche Zeit der höchsten Tumorrezidivwahrscheinlichkeit und auf Daten aus dem Organtranplantationsbereich berufen [13]. Jenseits der 5 Jahre wurde jegliche Biologikatherapie (damals v. a. TNF-In- 
hibioren) als sicher erachtet. Innerhalb der 5 Jahre wurde Rituximab der Vorzug gegeben, ohne dass dies zum damaligen Zeitpunkt durch Studien hätte begründet werden können - mit Ausnahme von CD20 + B-Zell-Neoplasien. Die einzige bis dato publizierte Studie aus dem hämatoonkologischen Bereich ergab sogar bei allen methodischen Problemen eher den Hinweis, dass Patienten die zusätzlich zu einer autologen Stammzelltransplantation Rituximab erhielten, ein höheres Risiko für Sekundärmalignome hatten als Patienten ohne Rituximabtherapie [14].

In der aktuellsten Version der ACR-Empfehlungen von 2015 wurde die Unterscheidung je nach Intervall zwischen Tumor- und Rheumatherapie aber aufgegeben. Bei Zustand nach einem soliden Malignom könne demnach die Basistherapie ohne Einschränkung wie bei RA-Patienten ohne Malignomanamnese behandelt werden. Ansonsten wurden für Hautkrebs und lymphoproliferative Erkrankungen Priorisierungen für DMARDs vorgenommen, die so sehr der Evidenz entbehren bzw. der damals schon verfügbaren, nicht-zitierten Evidenz zuwiderlaufen, dass sie hier nicht wiedergegeben werden sollen. Dass diese 2 malignen Krankheitsgruppen aber der besonderen Beachtung bedürfen, soll im Folgenden dargestellt werden.

\section{Direkte Evidenz zum Tumorrezidivrisiko unter rheumatologischer Therapie}

In nationalen Registern von RA-Patienten wurden Patienten mit Malignomanamnese im Sinne von Fall-Kontroll-Studien mit Patienten verglichen, die ähnliche Basistherapien hatten und keine maligne Erkrankung in der Vorgeschichte aufwiesen. Dabei wurde meist der Versuch unternommen, nach Risikofaktoren für eine Tumorrezidiv wie Krankheitsaktivität oder Adipositas zu adjustieren. Die ersten aussagekräftigen Daten stammten aus dem deutschen RABBIT-Register [15] und dem britischen Biologika-Register [16]. Darüber hinaus sind v. a. noch die Analysen des US-amerikanischen CORRONA-Registers [17], des dänischen DANBIO-Registers [18] und - erst kürzlich publiziert - des schwedischen ARTIS-Registers [19] zu nennen. Vor kurzem wurde eine Metanalyse von Xie und Kollegen über 11 Studien publiziert, die oben genannte Register miteinschließt [20]. Verwertbar sind die Daten lediglich für TNFInhbitoren und Rituximab. Erfreulicherweise zeigte sich jeweils kein erhöhtes Rezidivrisiko für TNFI-Inhibitoren oder Rituximab im Vergleich zu Patienten die konventionell-synthetische Basistherapeutika (csDMARDS) erhielten. Einschränkend ist zu bemerken, dass bei den meisten Studien das Intervall zwischen onkologischer- und rheumatologischer Therapie durchschnittlich deutlich mehr als 5 Jahre betrug - mit 2 Ausnahmen: Rituximab wurde im deutschen Register, der älteren ACR-Empfehlung folgend, schon früher eingesetzt und das dänische Register erlaubte einen Vergleich von Patienten mit einem Intervall bis zur TNF-Inhibitor-Therapie von mehr oder weniger als 5 Jahren. Hier zeigte sich numerisch sogar ein etwas geringeres Risiko für Tumorrezidive bei kürzerem Intervall selbst nach Adjustierung für initiale Tumorstadien. Allerdings zeigte sich hier, dass tatsächlich die am Register teilnehmenden Ärzte Biologika eher bei initial niedrigeren Tumorstadien einsetzten. Eine Metaanalyse von 16 Studien bei unterschiedlichen Autoimmunerkrankungen (RA, chronisch-entzündliche Darmerkrankungen und Psoriasis) konnten ebenfalls keinen statistisch signifikanten Unterschied in der Tumorrezidivinzidenz bei Intervallen von mehr oder weniger als 6 Jahren nachweisen [21]. Da die meisten Registerdaten aber im Durchschnitt lange Intervalle zwischen onkologischer und rheumatologischer Therapie aufweisen und Registerstudien einem erheblichen Bias unterliegen können, sollte trotzdem weiterhin die Länge des Intervalls in die rheumatologische Therapieentscheidung mit einbezogen werden.

Die wahrscheinlich aussagekräftigste Studie zum Tumorrezidivrisiko mit kurzem Intervall stellt die Analyse von US-amerikanischen Versicherungsdaten mit sehr großer Fallzahl von Mamtani und Kollegen dar [22]. Es handelte sich um Patientinnen mit rheumatoider Arthritis (>90\%) oder chronisch-entzündlichen Darmerkrankungen, die nach kurativer Operation eines Mammakarzinoms Methotrexat, Thiopurine, TNFI-Inhibitoren, Kombinationen davon oder keine Basistherapie erhielten. Bei mehr als $90 \%$ der Patientinnen wurden die TNF-Inhibtoren bereits innerhalb eines Jahres nach OP wieder begonnen und selbst bei kontinuierlicher Gabe der TNFIInhibitoren ließ sich kein erhöhtes Mammakarzinom-Rezidivrisiko feststellen. Allerdings betrug die Nachbeobachtung nur 3,4 Jahre. Diese Studie kann ein Stück weit als Gegenargument gegen die Einschätzung angeführt werden, dass in Bezug auf Biologikatherapie nach Mammakarzinom besondere Zurückhaltung zu wahren sei, weil Mammakarzinom-Rezidive auch deutlich später als 5 Jahre nach Operation auftreten können. Im britischen Register zeigte sich bei relativ großen Fallzahlen auch keine erhöhte Inzidenz von neuen Mammakarzinomen unter TNFI im Vergleich zu csDMARDS [23].

\section{Daten zu Einzelsubstanzen}

Die Datenlage zu allen in der Rheumatologie gängigen Substanzen detailliert darzustellen, würde im Rahmen dieses Übersichtsartikels zu weit führen. Darum soll im Folgenden die Datenlage stichpunktartig gemäß > Tab. 1 und das Tumorrisiko der Einzelsubstanzen oder Substanzgruppen zusammenfassend bewertet werden. Zitiert wird nur aktuelle Literatur. Für eine vertiefte Lektüre sei hier auf aussagekräftige Übersichtsarbeiten hingewiesen [24-27].

Gleich eingangs sei erwähnt, dass lediglich für Cyclophosphamid ein klares Signal für ein erhöhtes Tumorrisiko bzw. Tumorrezidivrisiko bei höheren Kumulativdosierungen besteht. Die sonst in der Rheumatologie üblichen immunmodulatorischen oder immun-

> Tab. 1 Bewertung der Evidenz zum Tumorrisiko immunmodulatorischer bzw. Immunsuppressiver Medikamente in der Rheumatologie.

\begin{tabular}{|l|l|}
\hline $\begin{array}{l}\text { Güte der } \\
\text { Evidenz }\end{array}$ & Definition \\
\hline Begrenzt & $\begin{array}{l}\text { Keine Sicherheitsdaten aus randomisiertem Studien- } \\
\text { programm bei rheumatisch-entzündlichen Erkrankun- } \\
\text { gen mit langer Nachbeobachtung }\end{array}$ \\
\hline Mäßig & $\begin{array}{l}\text { Sicherheitsdaten aus randomisiertem Studienpro- } \\
\text { gramm bei rheumatisch-entzündlichen Erkrankungen } \\
\text { mit langer Nachbeobachtung, keine Registerdaten }\end{array}$ \\
\hline Gut & $\begin{array}{l}\text { Zusätzlich aussagekräftige Registerdaten zum Risiko } \\
\text { inzidenter maligner Erkrankungen }\end{array}$ \\
\hline Sehr gut & $\begin{array}{l}\text { Zusätzlich aussagekräftige Daten aus Registern oder } \\
\text { Versicherungsdatenbanken zum Rezidivrisiko bei } \\
\text { vorbestehender maligner Erkrankung }\end{array}$ \\
\hline
\end{tabular}


- Tab. 2 Versuch einer Bewertung des Tumorrisikos von immunmodulatorischen und immunsuppressiven Therapien bezüglich Datenlage ( grün = gut, orange = mäßig, rot $=$ begrenzt $;$ siehe auch $\boldsymbol{\nabla}$ Tab. 1 ) und Hinweisen für Tumorrisiko aus der Literatur: grün= niedrigeres Risiko, orange $=$ gleiches Risiko oder rot $=$ höheres Risiko im Vergleich zu anderen Substanzen. Eine grenzwertiger Bewertung zu Datenlage und Risiko wurde durch Farbzwischentöne zum Ausdruck gebracht.

\begin{tabular}{|l|l|l|}
\hline Substanz & Daten & Risiko \\
\hline Methotrexat & & \\
\hline SSZ, HCQ & & \\
\hline Leflunomid & & \\
\hline CSA, Azathioprin & & \\
\hline Mycophenolat & & \\
\hline Cyclophosphamid & & \\
\hline TNF-Inhibitoren & & \\
\hline Rituximab & & \\
\hline Tocilizumab & \\
\hline Abatacept & \\
\hline Interleukin 1-Inhibition & \\
\hline Ustekinumab & \\
\hline Andere Biologika & \\
\hline JAK-Inhibitoren & \\
\hline Apremilast & \\
\hline
\end{tabular}

suppressiven Therapien dürften allenfalls einen geringen- in manchen Situationen sogar günstigen - Einfluss auf diese Risiken haben. Mit zunehmender Evidenz bestätigt sich eher dieses positive Bild.

In $\triangleright$ Tab. 2 wurde vor dem Hintergrund der relevanten Literatur der Versuch unternommen zu beurteilen, inwieweit sich Einzelsubstanzen der rheumatologischen Therapie auf das Risiko für das Auftreten von neuen Malignomen oder Malignomrezidiven auswirken könnten. Dies stellt zwar eine erhebliche Vereinfachung der komplexen Thematik dar, soll aber als schnelle Orientierung und als Ausgangspunkt für weitere Diskussion und Recherche dienen. Das Gesamturteil über eine Substanz sollte sich immer sowohl auf die Güte der Datenlage als auch auf die daraus hervorgehenden Hinweis zum Malignomrisiko beziehen. So würde man sich im Zweifel bei alternativen Substanzen mit niedrigem Risiko eher für das mit der besseren Datenlage entscheiden.

\section{Methotrexat}

Datenlage: gut. Methotrexat wird als Erhaltungstherapie bei akuten lymphatischen Leukämien eingesetzt. Es muss von einem Kumulativdosis-abhängig leicht erhöhten Risiko für nicht-melanozytären Hautkrebs ausgegangen werden, was sich auch in der großen randomisierten Studie zum Einsatz von Methotrexat bei kardiovaskulären Risikopatienten nochmals zeigte [28, 29]. Die v. a. von japanischen Autoren beschriebene Häufung lymphoproliferativer Erkrankungen, die zuweilen nach Absetzen von Methotrexat spontan remittieren [30], bestätigt sich in der Zusammenschau sonstiger ausführlicher Registerdaten nicht [31].

\section{Leflunomid, Hydroxychloroquin, Sulfasalazin}

Datenlage: mäßig für Leflunomid, schlecht für Hydroxychloroquin und Sulfasalazin. Leflunomid besitzt zumindest in vitro tumorinhibierende Effekte. Die numerische Häufung von Pankreaskarzinomen im RABBIT-Register unter Leflunomid wurde in anderen Registern nicht gefunden [32].

Für Hydroxychloroquin suggeriert eine Studie bei SLE und mit geringen Fallzahlen maligner Ereignisse einen protektiven Effekt gegen maligne Erkrankungen [33]. Eine neuere Studie zu Kollagenosen lässt lediglich einen günstigen Effekt auf die Häufigkeit metastasierter Tumorerkranken vermuten [34]. Onkologische Therapien in Kombination mit Hydroxychloroquin werden aufgrund des Effektes auf Autophagozytose diskutiert.

Sulfasalazin könnte ähnlich wie Mesalazin das Risiko für Kolonkarzinome bei Colitis ulcerosa reduzieren [35].

\section{Apremilast}

Datenlage: mäßig. FDA-Nebenwirkungsberichte lassen vermuten, dass Apremilast ein noch geringeres Tumorrisiko als Ustekinumab aufweist [36]. Ähnlich wie bei Hydroxychloroquin und Sulfasalazin erscheint ein ungünstiger Effekt auf Tumorentstehung schon allein aufgrund des Wirkmechanismus für unwahrscheinlich.

\section{Azathioprin, Ciclosporin A, Mycophenolat}

Datenlage: mäßig. Es existieren Hinweise, dass es unter kombinierter Therapie von Azathioprin mit Infliximab bei Männern mit chronisch-entzündlicher Darmerkrankung zu gehäuftem Auftreten der sehr seltenen hepatosplenischen T-Zell-Lymphome kommen könnte [37]. Die in einer kürzlich publizierten Studie postulierte Häufung von myeloischen Neoplasien durch Azathioprin bei unterschiedlichen Autoimmunerkrankungen wurde in anderen Studien nicht gefunden und ist wahrscheinlich der Tatsache geschuldet, dass sehr heterogene Populationen verglichen wurden [38]. Sowohl für Azathioprin als auch für Ciclosporin A erscheint das Risiko für NMSC erhöht zu sein [39], bei CSA v. a. in Zusammenhang mit Lichttherapie bei Psoriasis [40]. Die Daten über Azathioprin aus unterschiedlichen Registern zur Inzidenz von Krebs insgesamt, von Urothelkarzinomen und Lymphomen ist widersprüchlich, auch wenn sich kein starkes Signal ergibt. Für MMF existieren v. a. Studien aus dem Organtransplantationsbereich. Hier scheint das Risiko für Krebs insgesamt für Hauttumore und für Post-Transplantationslymphoproliferativen Erkrankungen im Vergleich zu anderen immunsuppressiven Regimen nach Transplantation etwas niedriger zu sein [41].

\section{Cyclophosphamid}

Datenlage: gut. Abhängig von der Kumulativdosis von Cyclophosphamid ist das Risiko für das Neuauftreten von Malignomen oder für Tumorrezidive erhöht. Die meisten Studien geben als Grenze, ab der dieses Risiko signifikant ansteigt, Werte zwischen 20 und 30 $\mathrm{g}$ an [42]. Eine Studie über SLE-Patientinnen in Südkorea fand sogar bereits ein erhöhtes Risiko für inzidente Malignome bei einer Kumulativdosis von $>6 g$ [43]. Selbst dieser Wert wird aber durch moderne Cyclophosphamidprotokolle wie das Euro-Lupus-Protokoll nicht erreicht. 


\section{Biologika}

\section{TNF-Inhibitoren}

Datenlage: sehr gut. Es ist ein leicht erhöhtes Risiko für NMSC zu vermuten [44]. Ob ein erhöhtes Risiko für maligne Melanome unter TNFI besteht, wurde lange Zeit kontrovers diskutiert ohne dass sich klare Signale ergaben. Eine Analyse von 11 europäischen RA-Registern [45] und die aktuellste Analyse des australischen ARAD-Registers [46] für RA-Patienten ergaben keinen Hinweis auf eine Risikoerhöhung. Mehrere Studien ergaben kein signifikant erhöhtes Risiko für eine Progression von bekannten zervikalen intraepithelialen Dyspalsien in Zervixkarzinome unter TNFI [47]. In einer aktuellen Auswertung von US-amerikanischen Versicherungsdaten wird ein erhöhtes Lymphomrisiko vermutet [48], das sich in Studien aus mehreren nationalen Registern nicht fand [49]. Die wichtigen und beruhigenden Daten zu Studien zum Tumorrezidivrisiko unter TNFI wurden weiter oben dargestellt. Interessant sind in diesem Zusammenhang Daten aus dem Bereich der immunonkologischen Therapien mit sogenannten Checkpointinhibitoren (ICPI): Mausmodelle [50,51] und eine erste Fallserie [52] lassen vermuten, dass die Kombination von ICPI mit TNFI nicht nur zu einer geringeren Rate von immunvermittelten Nebenwirkungen, sondern auch zu einer besseren Tumorkontrolle führen könnte.

\section{Rituximab}

Datenlage: sehr gut. Obwohl Rituximab gerade in Deutschland schon lange den alten ACR-Empfehlungen folgend bereits relativ kurz nach kurativer Tumortherapie eingesetzt wird, finden sich in der Literatur zu rheumatologischen Krankheitsbildern (v. a. zur RA) keine Hinweise auf ein erhöhtes Risiko für Tumorrezidive oder für neue Malignome. Die günstigen Registerdaten zum Tumorrezidivrisiko wurden oben präsentiert.

\section{Tocilizumab, Sarilumab}

Datenlage: gut für Tocilizumab, begrenzt für Sarilumab. Tocilizumab findet in unterschiedlichen Therapiesituationen in der Onkologie therapeutische Verwendung: Behandlung des Morbus Castleman (Zulassung in Japan), Behandlung des Zytokinsturms nach CAR-T-ZellTherapie (Zulassung), Behandlung von immunvermittelten Nebenwirkungen von Checkpointinhibitoren (Fallserien, Expertenmeinung) [53]. Die Überlegungen zum Wirkmechanismus, die Tocilizumab im Zusammenhang mit malignen Erkrankungen in einem günstigen Licht erscheinen lassen, sollten nicht überinterpretiert werden. Eine große Analyse von US-amerikanischen Versicherungsdatenbanken fand zumindest kein höheres Risiko für maligne Ereignisse bei RA unter Tocilizumab im Vergleich zu TNFI oder Abatacept, wobei NMSC von der Analyse ausgeschlossen waren [54].

\section{Abatacept}

Datenlage: gut. Auch hier sollten Überlegungen zum Wirkmechanismus, der dem des Checkpointinhibitors Ipilimumab genau entgegengesetzt ist, nicht überinterpetiert werden. Allerdings sprechen eine große Registerstudie [55] und eine Analyse von US-amerikanischen Versicherungsdaten [56] für ein erhöhtes Risiko für NMSC selbst im Vergleich zu anderen Biologika. Für Krebsinzidenz insgesamt und Melanominzidenz sind die Daten widersprüchlich [57].

\section{Secukinumab, Ixekizumab}

Datenlage: mäßig für Secukinumab, begrenzt für Ixekizumab. IL17Signalwege können theoretisch sowohl tumorfördernd, als auch tumorinhibierend sein. Die Argumentation der deutschen S3-Leitlinie zur Behandlung der Psoriasis, die Secukinumab und Ustekinumab bei maligner Vorerkrankung TNFI gegenüber den Vorzug gibt, erscheint nicht schlüssig in Bezug auf Secukinumab [58]. Daten zur angeblichen Erhöhung des Tumorrisikos unter TNFI werden hier überinterpretiert und Daten zu Secukinumab gegenübergestellt, die zwar keine Hinweise auf erhöhte Krebsinzidenz ergeben, aber zur Untersuchung dieser Fragestellung aufgrund geringer Fallzahlen und kurzer Nachbeobachtung auch nicht geeignet sind.

\section{Ustekinumab}

Datenlage: gut. In einer Auswertung des PSOLAR-Registers zur Therapie der Psoriasis wiesen Ustekinumab und Methotrexat ein niedrigeres Krebsrisiko insgesamt im Vergleich zu TNFi auf [59]. Dies würde die oben erwähnte Empfehlung in der deutschen PsoriasisLeitlinie unterstützen, sollte aber idealerweise in anderen Studien und Therapiesituationen bestätigt werden, zumal die bereits erwähnten, schwierig zu interpretierenden Daten von FDA-Nebenwirkungsberichten für ein höheres Risiko im Vergleich zu Apremilast sprechen.

\section{Anakinra, Canakinumab}

Datenlage: mäßig bis gut. Anakinra wird zur Therapie des Schnitzler-Syndroms eingesetzt. Bei dieser Erkrankung besteht eine Assoziation der Urtikariavaskulitis zur monoklonalen Gammopathie unklarer Signifikanz, die eine Präkanzerose darstellt. In der CANTOSStudie erhielten Patienten mit erhöhtem CRP nach Myokardinfarkt entweder Canakinumab oder Placebo [60]. Trotz Erreichen des primären Endpunktes führte diese Studie zwar nicht zur Zulassung in dieser Indikation, allerdings fiel eine geringere Inzidenz für Bronchialkarzinome im Studienarm auf. Tatsächlich kann Interleukin-1 tumorfördernde Effekte haben. Diese Beobachtung wird in einem onkologischen Studienprogramm weiterverfolgt.

\section{Belimumab}

Datenlage: mäßig. Gesonderte Erkenntnisse zum Risiko für maligne Erkrankungen liegen nicht vor.

\section{JAK-Inhibitoren}

Datenlage: gut für Tofacitinib, mäßig für Baricitinib, begrenzt für Upadacitinib. Die Betrachtung des Tumorrisikos von JAK-Inhibitoren sollte jetzt und in Zukunft besonders fundiert erfolgen, da es sich um hochpotent immunsuppressive Medikamente handelt und bereits jetzt gewisse Signale zu verzeichnen sind, die aber noch kein eindeutiges Bild liefern. Patienten mit Myeloproliferativen Neoplasien (MPN) haben gehäuft monoklonale B-Zell-Populationen im Knochenmark und damit ein höheres Risiko für B-Zell-Neoplasien. Eine hämatoonkologische Arbeitsgruppe aus Wien wertete eine Kohorte mit MPN-Patienten aus, von denen ein Teil mit unterschiedlichen zugelassenen und experimentellen JAK-Inhibitoren behandelt wurde [61]. In dieser Kohorte zeigte sich ein signifikant erhöhtes Risiko für aggressive B-Zellneoplasien unter JAK-Inhibitoren. Diese Beobachtung wurde durch ein Mausmodell der Arbeits- 
gruppe unterstützt, aber nicht durch eine analoge Untersuchung einer größeren MPN-Kohorte aus dem MD Anderson bestätigt [62]. Im randomisierten RA-Studienprogramm von Upadacitinib fand sich unter der nicht zugelassenen Dosis 30 mg ein signifikant höheres Risiko für NMSC als unter 15 mg [63]. Auch bei Baricitinib traten unter der höheren Dosis $4 \mathrm{mg}$ bei RA numerisch relativ mehr NMSCs auf als unter $2 \mathrm{mg}$ [64]. Nur für Tofacitinib existieren bereits aussagekräftige Registerdaten. Im US-amerikanischen CORRONARegister waren die Raten für NMSC und für Krebs ohne NMSC unter Tofacitinib mit den Biologika vergleichbar und unterschieden sich nicht signifikant [65]. Allerdings sei nochmals auf die Möglichkeit des Bias in Registern verwiesen. Auch ist diese Studie noch nicht vollpubliziert.

\section{Therapiestrategien abhängig von der Situation}

Vor dem Hintergrund dieser Betrachtungen soll im Folgenden der Versuch unternommen werden, Vorschläge zur rheumatologischen Therapie von Patienten mit Malignomanamnese zu formulieren. Diese Vorschläge sind eine Synthese aus bereits bestehenden Empfehlungen in Leitlinien und der geschilderten und gesichteten Evidenz. Sie unterlagen keinem Konsensus-Prozess und geben letztlich die individuelle Meinung des Autors wieder:

- Therapeutische Entscheidungen sind in diesem Zusammenhang emotional sehr beladen und müssen mit dem Patienten und dem onkologisch behandelnden Kollegen ausführlich besprochen werden.

- In einer palliativen Therapiesituation muss unterschieden werden, ob die Prognose und onkologische Therapieoptionen sehr begrenzt sind oder ob die Möglichkeit einer langfristigen Krankheitskontrolle durch (moderne) onkologische Therapiekonzepte besteht. Insbesondere die Bewertung von DMARDTherapien nach immunoonkologischen Therapien fällt schwer, kann aber durch onkologische Erfahrungen zur immunsuppressiven Therapie immunvermittelter Nebenwirkungen dieser Behandlungen unterstützt werden. Wenn das primäre Therapieziel Symptomlinderung ist, kann die rheumatologische Therapie meist ohne Einschränkung betrieben werden. Beim Ziel einer langfristigen Tumorkontrolle sollte man sich ähnlich wie bei kurativen Therapiekonzepten verhalten.

- Bei kurativem Therapieziel kann man rheumatologische Therapie 5 Jahre nach Abschluss der onkologischen Therapie wahrscheinlich ohne Einschränkung durchführen. Mehrjährige Erhaltungstherapien sollten dieses Intervall nicht noch mehr hinauszögern. Auch jenseits eines Intervalles von 5 Jahren sollte man bei Malignem Melanom Abatacept, JAK-Inhibitoren und vielleicht auch TNF-Inhibitoren aufgrund der unklaren Datenlage eher meiden, v. a. wenn alternative Therapieoptionen existieren.

- Bei kurativ behandelten Lymphomen sollte man mit JAK-Inhibitoren und vielleicht auch TNF-Inhibitoren eher zurückhaltend sein. Dagegen wirken sich Rituximab und vielleicht auch Tocilizumab bei stattgehabten B-Zell-Neoplasien oder Plasmazelldyskrasien günstig aus.

- Bei kurativem Therapieziel empfiehlt sich innerhalb des 5 Jahresintervalls :
- Pausieren der Basistherapie und Überbrückung mit Glukokortikosteroiden (bzw. Nicht-steroidalen Antirheumatika) während Chemotherapie

- Beachten von Wechselwirkungen bei langfristigen onkologischen Erhaltungstherapien

- Ansonsten eine konsequente Basistherapie im Sinne eines abgestuften Konzeptes, das sich an individuellem Tumorrezidivrisiko und Datenlage zu den in Frage kommenden DMARDs orientiert. Die Datenlage ist sowohl danach zu bewerten, wie viele Studien in welcher Qualität vorliegen, als auch danach, ob sich Hinweise für geringeres oder höheres Tumorrisiko im Vergleich zu anderen Substanzen gezeigt haben.

- Langfristig unkontrollierte Entzündung oder langfristig hochdosierte Glukokortikoidtherapie durch übermäßige Zurückhaltung vor Basistherapie sollte aber unbedingt vermieden werden.

- Aufgrund der hohen Komplexität der Wechselwirkungen von Immunsystem und Tumor in Zusammenhang mit immunonkologischen Therapien (Checkpointinhibitoren) empfiehlt es sich rheumatologische Therapieentscheidungen von einem spezialisierten Zentrum treffen zu lassen.

\section{FAZIT FÜR DIE PRAXIS:}

- Die Entscheidung über immunmodulierende oder immunsuppressive Therapien bei Patienten mit Malignomanamnese muss in der Regel interdisziplinär und stets in enger Absprache mit dem Patienten getroffen werden.

- Eine zunehmende Datenfülle hilft bei der Beurteilung der DMARD-Einzelsubstanzen. Die Datenlage ist aufgrund unterschiedlicher Einschränkungen der Studienformate aber komplex zu beurteilen, ergibt aber bis auf ganz wenige Ausnahmen ein eher positives Bild.

- Zu wenigen Substanzen (TNF-Inhibitoren, Rituximab) existieren Studien, die primär das Tumorrezidivrisiko bei stattgehabter maligner Erkrankung untersucht haben. Bei anderen Substanzen kann man sich durch Daten über das Risiko inzidenter Tumore und begrenzt durch Betrachtung präklinischer Modelle informieren.

- Nur zu Cyclophophosphamid in hoher Kumulativdosis besteht ein starkes Signal für ein erhöhtes Tumorrisiko. Bei vorhandenen Alternativen sollte man wahrscheinlich Abatacept, JAK-Inhibitoren und vielleicht in manchen Situationen auch TNF-Inhibitoren eher meiden. Für TNF-Inhibitoren ist die Datenlage in bestimmten Situationen (z. B. Zustand nach Mammakarzinom) besonders aussagekräftig und erscheint dort eher günstig.

- Mehrere Substanzen erhöhen das Risiko für nicht-melanozytäre Hautkrebsformen. Diesem Risiko kann gut durch Veranlassung eines konsequenten Hauttumor-Screenings begegnet werden. 


\section{Interessenkonflikt}

Berater-/Gutachtertätigkeit: Chugai/Roche, Hexal/Sandoz, Gilead, AbbVie, Janssen-Cilag, Boehringer/Ingelheim

Vortrags-/Schulungstätigkeit: Novartis, AbbVie, Chugai/Roche, Janssen-Cilag

Kongresse: Chugai/Roche, Boehringer/Ingelheim, Celgene, Medac

\section{Literatur}

[1] Baecklund E, Iliadou A, Askling J et al. Association of chronic inflammation, not its treatment, with increased lymphoma risk in rheumatoid arthritis. Arthritis Rheum 2006; 54: 692-701

[2] Zhang $Y$, Sun $Y$, Zhang Q. Prognostic value of the systemic immuneinflammation index in patients with breast cancer: a meta-analysis. Cancer Cell Int 2020; 20: 224

[3] Hirano T, Hirayama D, Wagatsuma K et al. Immunological Mechanisms in Inflammation-Associated Colon Carcinogenesis. Int J Mol Sci 2020; 21: 3062

[4] Moik F, Zöchbauer-Müller S, Posch F et al. Systemic Inflammation and Activation of Haemostasis Predict Poor Prognosis and Response to Chemotherapy in Patients with Advanced Lung Cancer. Cancers (Basel) 2020; 12: E1619

[5] Kampan NC, Madondo MT, McNally OM et al. Interleukin 6 Present in Inflammatory Ascites from Advanced Epithelial Ovarian Cancer Patients Promotes Tumor Necrosis Factor Receptor 2-Expressing Regulatory T Cells. Front Immunol 2017; 8: 1482

[6] Krathen MS, Gottlieb AB, Mease P]. Pharmacologic immunomodulation and cutaneous malignancy in rheumatoid arthritis, psoriasis, and psoriatic arthritis. J Rheumatol 2010; 37: 2205-2215

[7] Mercer LK, Davies R, Galloway JB et al. Risk of cancer in patients receiving non-biologic disease-modifying therapy for rheumatoid arthritis compared with the UK general population. Rheumatology 2013; 52: 9198

[8] Nocturne G, Mariette X. Sjögren Syndrome-associated lymphomas: an update on pathogenesis and management. $\mathrm{Br}$ J Haematol 2015; 168 : 317-327

[9] Feldman CH, Liu J, Feldman S et al. Risk of high-grade cervical dysplasia and cervical cancer in women with systemic lupus erythematosus receiving immunosuppressive drugs. Lupus 2017; 26: 682-689

[10] Lopez-Olivo MA, Colmegna I, Karpes Matusevich AR et al. Systematic Review of Recommendations on the Use of Disease-Modifying Antirheumatic Drugs in Patients With Rheumatoid Arthritis and Cancer. Arthritis Care Res (Hoboken) 2020; 72: 309-318

[11] Singh JA, Furst DE, Bharat A et al. 2012 update of the 2008 American College of Rheumatology recommendations for the use of diseasemodifying antirheumatic drugs and biologic agents in the treatment of rheumatoid arthritis. Arthritis Care Res (Hoboken) 2012; 64: 625-639

[12] Singh JA, Saag KG, Bridges SL Jr et al. 2015 American College of Rheumatology Guideline for the Treatment of Rheumatoid Arthritis. Arthritis Care Res (Hoboken) 2016; 68: 1-25

[13] Penn I. Evaluation of transplant candidates with pre-existing malignancies. Ann Transplant 1997; 2: 14-17

[14] Tarella C, Passera R, Magni M et al. Risk factors for the development of secondary malignancy after high-dose chemotherapy and autograft, with or without rituximab: a 20-year retrospective follow-up study in patients with lymphoma. J Clin Oncol 2011; 29: 814-824
[15] Strangfeld A, Hierse F, Rau R et al. Risk of incident or recurrent malignancies among patients with rheumatoid arthritis exposed to biologic therapy in the German biologics register RABBIT. Arthritis Res Ther 2010; 12: R5

[16] Dixon WG, Watson KD, Lunt M et al. Influence of antitumor necrosis factor therapy on cancer incidence in patients with rheumatoid arthritis who have had a prior malignancy: results from the British Society for Rheumatology Biologics Register. Arthritis Care Res 2010; 62: 755-763

[17] Pappas DA, Rebello S, Liu M et al. Therapy with Biologic Agents After Diagnosis of Solid Malignancies: Results from the Corrona Registry. J Rheumatol 2019; 46: 1438-1444

[18] Dreyer L, Cordtz RL, Hansen IM] et al. Risk of second malignant neoplasm and mortality in patients with rheumatoid arthritis treated with biological DMARDs: a Danish population-based cohort study. Ann Rheum Dis 2018; 77: 510-514

[19] Raaschou P, Sö derling J, Turesson C et al. ARTIS Study Group. Tumor necrosis factor inhibitors and cancer recurrence in Swedish patients with rheumatoid arthritis: a nationwide population-based cohort study. Ann Intern Med 2018; 169: 291-299

[20] Xie W, Xiao S, Huang Y et al. A meta-analysis of biologic therapies on risk of new or recurrent cancer in patients with rheumatoid arthritis and a prior malignancy. Rheumatology (Oxford). 2019; Oct 17. [Epub ahead of print]

[21] Shelton E, Laharie D, Scott Fl et al. Cancer Recurrence Following Immune-Suppressive Therapies in Patients With Immune-Mediated Diseases: A Systematic Review and Meta-analysis. Gastroenterology 2016; 151: 97-109.e4

[22] Mamtani R, Clark AS, Scott Fl et al. Association between breast cancer recurrence and immunosuppression in rheumatoid arthritis and inflammatory bowel disease: a cohort study. Arthritis Rheumatol 2016; 68: 2403_11

[23] Mercer LK, Lunt M, Low AL et al. Risk of solid cancer in patients exposed to anti-tumour necrosis factor therapy: results from the British Society for Rheumatology Biologics Register for Rheumatoid Arthritis. Ann Rheum Dis 2015; 74: 1087-1093

[24] Schmalzing M, Strangfeld A, Tony HP. Medikamentöse Therapie der rheumatoiden Arthritis bei Malignomanamnese. Epidemiologische Daten [Medication treatment of rheumatoid arthritis with a history of malignancy. Epidemiological data]. Z Rheumatol 2016; 75: 22-31

[25] Regierer AC, Strangfeld A. Rheumatoid arthritis treatment in patients with a history of cancer. Curr Opin Rheumatol 2018; 30: 288-294

[26] Shelton E, Laharie D, Scott Fl et al. Cancer Recurrence Following Immune-Suppressive Therapies in Patients With Immune-Mediated Diseases: A Systematic Review and Meta-analysis. Gastroenterology. 2016; 151: 97-109.e4

[27] De Cock D, Hyrich K. Malignancy and rheumatoid arthritis: Epidemiology, risk factors and management. Best Pract Res Clin Rheumatol 2018; 32: 869-886

[28] Lange E, Blizzard L, Venn A et al. Disease-modifying anti-rheumatic drugs and non-melanoma skin cancer in inflammatory arthritis patients: a retrospective cohort study. Rheumatology (Oxford) 2016; 55: $1594-1600$

[29] Solomon DH, Glynn R], Karlson EW et al. Adverse Effects of Low-Dose Methotrexate: A Randomized Trial. Ann Intern Med. 2020; Feb 18. [Epub ahead of print]

[30] Rizzi R, Curci P, Delia M et al. Spontaneous remission of "methotrexate-associated lymphoproliferative disorders" after discontinuation of immunosuppressive treatment for autoimmune disease. Review of the literature. Med Oncol 2009; 26: 1-9

[31] Hellgren K, Baecklund E, Backlin C et al. Rheumatoid Arthritis and Risk of Malignant Lymphoma: Is the Risk Still Increased? Arthritis Rheumatol 2017; 69: 700-708 
[32] Behrens F, Koehm M, Burkhardt H. Update 2011: leflunomide in rheumatoid arthritis - strengths and weaknesses. Curr Opin Rheumatol 2011; 23: 282-287

[33] Ruiz-Irastorza G, Ugarte A, Egurbide MV et al. Antimalarials may influence the risk of malignancy in systemic lupus erythematosus. Ann Rheum Dis 2007; 66: 815-817

[34] Fardet L, Nazareth I, Petersen I. Effects of chronic exposure of hydroxychloroquine/chloroquine on the risk of cancer, metastasis, and death: a population-based cohort study on patients with connective tissue diseases. Clin Epidemiol 2017; 9: 545-554

[35] Lopez A, Pouillon L, Beaugerie L et al. Colorectal cancer prevention in patients with ulcerative colitis. Best Pract Res Clin Gastroenterol 2018, 32-33: 103-109

[36] T. J. Moore Safety perspectives: cancer risks of biological products for psoriasis. https://www.ismp.org/quarterwatch/safety-perspectives (accessed: March 29, 2018)

[37] Thai A, Prindiville T. Hepatosplenic T-cell lymphoma and inflammatory bowel disease. J Crohns Colitis 2010; 4: 511-22

[38] Ertz-Archambault N, Kosiorek H, Taylor GE et al. Association of Therapy for Autoimmune Disease With Myelodysplastic Syndromes and Acute Myeloid Leukemia. JAMA Oncol 2017; 3: 936-943

[39] Scott Fl, Mamtani R, Brensinger CM et al. Risk of Nonmelanoma Skin Cancer Associated With the Use of Immunosuppressant and Biologic Agents in Patients With a History of Autoimmune Disease and Nonmelanoma Skin Cancer. JAMA Dermatol 2016; 152: 164-172

[40] Muellenhoff MW, Koo JY. Cyclosporine and skin cancer: an international dermatologic perspective over 25 years of experience. A comprehensive review and pursuit to define safe use of cyclosporine in dermatology. J Dermatolog Treat 2012; 23: 290-304

[41] Vos M, Plasmeijer El, van Bemmel BC et al. Azathioprine to mycophenolate mofetil transition and risk of squamous cell carcinoma after lung transplantation. J Heart Lung Transplant 2018; 37: 853-859

[42] Hellbacher E, Hjorton K, Backlin C et al. Malignant lymphoma in granulomatosis with polyangiitis: subtypes, clinical characteristics and prognosis. Acta Oncol 2019; 58: 1655-1659

[43] Kang KY, Kim HO, Yoon HS et al. Incidence of cancer among female patients with systemic lupus erythematosus in Korea. Clin Rheumatol 2010; 29: 381-388

[44] Raaschou P, Simard JF, Asker Hagelberg C et al.ARTIS Study Group Rheumatoid arthritis, anti-tumour necrosis factor treatment, and risk of squamous cell and basal cell skin cancer: cohort study based on nationwide prospectively recorded data from Sweden. BMJ 2016; 352: i262

[45] Mercer LK, Askling J, Raaschou P et al. Risk of invasive melanoma in patients with rheumatoid arthritis treated with biologics: results from a collaborative project of 11 European biologic registers. Ann Rheum Dis 2017; 76: 386-391

[46] Staples MP, March L, Hill C et al. Malignancy risk in Australian rheumatoid arthritis patients treated with anti-tumour necrosis factor therapy: an update from the Australian Rheumatology Association Database (ARAD)prospective cohort study. BMC Rheumatol 2019; 3: 1

[47] Cordtz R, Mellemkjær L, Glintborg B et al. Malignant progression of precancerous lesions of the uterine cervix following biological DMARD therapy in patients with arthritis. Ann Rheum Dis 2015; 74: 1479-80

[48] Calip GS, Patel PR, Adimadhyam S et al. Tumor necrosis factor-alpha inhibitors and risk of non-Hodgkin lymphoma in a cohort of adults with rheumatologic conditions. Int J Cancer 2018; 143: 1062-1071

[49] Mercer LK, Galloway JB, Lunt M et al. Risk of lymphoma in patients exposed to antitumour necrosis factor therapy: results from the British Society for Rheumatology Biologics Register for Rheumatoid Arthritis. Ann Rheum Dis 2017; 76: 497-503
[50] Perez-Ruiz E, Minute L, Otano I et al. Prophylactic TNF blockade uncouples efficacy and toxicity in dual CTLA-4 and PD-1 immunotherapy. Nature 2019; 569: 428-432

[51] Bertrand F, Montfort A, Marcheteau E et al. TNF $\alpha$ blockade overcomes resistance to anti-PD-1 in experimental melanoma. Nat Commun 2017; 8: 2256

[52] Badran YR, Cohen JV, Brastianos PK et al. Concurrent therapy with immune checkpoint inhibitors and TNF $\alpha$ blockade in patients with gastrointestinal immune-related adverse events. J Immunother Cancer 2019; 7: 226

[53] Kim ST, Tayar ], Trinh VA et al. Successful treatment of arthritis induced by checkpoint inhibitors with tocilizumab: a case series. Ann Rheum Dis 2017; 76: 2061-2064

[54] Kim SC, Pawar A, Desai R] et al. Risk of malignancy associated with use of tocilizumab versus other biologics in patients with rheumatoid arthritis: A multi-database cohort study. Semin Arthritis Rheum 2019; 49: $222-228$

[55] Wadström H, Frisell T, Askling J. Anti-Rheumatic Therapy in Sweden (ARTIS)Study Group. Malignant Neoplasms in Patients With Rheumatoid Arthritis Treated With Tumor Necrosis Factor Inhibitors, Tocilizumab, Abatacept, or Rituximab in Clinical Practice: A Nationwide Cohort Study From Sweden. JAMA Intern Med 2017; 177: 1605-1612

[56] Montastruc F, Renoux C, Dell'Aniello S et al. Abatacept initiation in rheumatoid arthritis and the risk of cancer: a population-based comparative cohort study. Rheumatology (Oxford) 2019; 58: 683-691

[57] de Germay S, Bagheri H, Despas F et al. Abatacept in rheumatoid arthritis and the risk of cancer: a world observational post-marketing study. Rheumatology (Oxford). 2019; Dec 27, [Epub ahead of print]

[58] Nast A, Amelunxen LAugustin et al. S3 - Leitlinie zur Therapie der Psoriasis vulgaris Update. 2017; AWMF-Register Nr. 013/001

[59] Fiorentino D, Ho V, Lebwohl MG et al. Risk of malignancy with systemic psoriasis treatment in the Psoriasis Longitudinal Assessment Registry. J Am Acad Dermatol 2017; 77: 845-854.e5

[60] Ridker PM, MacFadyen JG, Thuren T et al.CANTOS Trial Group Effect of interleukin- $1 \beta$ inhibition with canakinumab on incident lung cancer in patients with atherosclerosis: exploratory results from a randomised, double-blind, placebo-controlled trial. Lancet 2017; 390: 1833-1842

[61] Porpaczy E, Tripolt S, Hoelbl-Kovacic A et al. Aggressive B-cell lymphomas in patients with myelofibrosis receiving JAK $1 / 2$ inhibitor therapy. Blood 2018; 132: 694-706

[62] Pemmaraju N, Kantarjian H, Nastoupil L et al. Characteristics of patients with myeloproliferative neoplasms with lymphoma, with or without JAK inhibitor therapy. Blood 2019; 133: 2348-2351

[63] Integrated safety summary, Abbvie data on file

[64] Smolen JS, Genovese MC, Takeuchi T et al. Safety Profile of Baricitinib in Patients with Active Rheumatoid Arthritis with over 2 Years Median Time in Treatment. J Rheumatol 2019; 46: 7-18

[65] Kremer J, Bingham C, Cappelli L et al. Comparison of Malignancy and Mortality Rates Between Tofacitinib and Biologic DMARDs in Clinical Practice: Five-Year Results from a US-Based Rheumatoid Arthritis Registry [abstract]. Arthritis Rheumatol 2019; 71: (suppl 10) https:// acrabstracts.org/abstract/comparison-of-malignancy-and-mortalityrates-between-tofacitinib-and-biologic-dmards-in-clinical-practicefive-year-results-from-a-us-based-rheumatoid-arthritis-registry/. Accessed October 6, 2020. 